\title{
NOUVELLE MÉTHODE POUR LA DÉTERMINATION DU RESTE DE LA FORMULE DE TAYLOR.
}

par LE D'. ANT. WINCKLER.

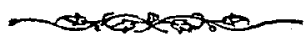

La détermination du reste de la formule de Taylor, qui a occupé d'abord d'Alembert et qui plus tard a été trouvée de différentes manières par Lagrange, Ampère et Cauchy, doil résulter immédiatement du développement de la série, en sorte, qu'on n'y a besoin que de la définition du quotient différentiel; car ce n'est qu'alors qu'on peut mettre en évidence cetie formule déja dans les éléments, pour en faire application plus tard à la solution d'un grand nombre de questions; et pour faire voir les grand avantages qu'elle y fournit.

Dans toutes les mélhodes connues la détermination du reste est ou trop prolixe, ou on n'en parle que bien tard dans l'exposition du calcul différentiel, - bien souvent en supposant la forme des membres de la série, - ou enfin, on la remet jusqu'au calcul intégral, de manière que les cas, où l'on aurait pu faire les applications les plus importantes de cette formule, sont dejà déterminés tous par des méthodes plus compliquées.

La méthode suivante satisfera peut-être mienx aux conditions susdites, en developpant en mème temps la série el son reste d'une manière, qui s'offre, pour ainsi dire de soi mème et qui n'exige que la connaissance des trois corollaires suivants:

A. Une fonction croît ou decroît avec sa variable croissante, selon que la valeur de son quotient différentiel est positive ou négative, ou, ce qui revient au même, la différence $\varphi(x+y)-\varphi(x)$ est de même signe que $\varphi^{\prime}(x)$, - pourvu que $\varphi^{\prime}(x)$ ne change pas de signe pour des valeurs entre les limites $x$ el $x+y$ de la variable.

B. Le quotient différentiel $f^{(i)}(x)$ ne peut ètre fini et continu dans l'intervalle de $x$ à $x+y$ de la variable, si ne sont aussi les quotients précédents $f^{(n-1)}(x)$, $f^{(n-2)}(x) \ldots f^{\prime}(x), f(x)$.

C. L'équation $\frac{\mathrm{d} f(x+y)}{\mathrm{d} x}=\frac{\mathrm{d} f(x+y)}{\mathrm{d} y}$ a toujours lieu, quand on suppose finie el continue la fonction $f(x+y)$ pour toutes les valeurs de $x$ jusqu'a $x+y$.

1.

Le développement désiré de la fonction $f(x+y)$ jusqu'à la puissance $n^{\text {me de }}$ Tom. II. N: $3 . ; 859$. 
l'acroissement $y$ de la variable soit :

$$
f(x+y)=\mathrm{X}_{0}+\mathrm{X}_{\mathrm{r}} y+\mathrm{X}_{2} y^{2}+\ldots+\mathrm{X}_{n-2} y^{n-2}+\mathrm{X}_{n-1} y^{n-1}+\mathrm{U},
$$

$X_{0}, X_{1}, \ldots X_{n-x}$ étant des fonctions de la seule variable $x$; et soil $U$ le reste, fonction de $x$ et $y$, que nous allons déterminer.

En différentiant par rapport à $x$, et puis par rapport à $y$, nous aurons l'équation:

$$
\begin{aligned}
& \mathbf{X}_{0}^{\prime}+y \mathbf{X}_{1}^{\prime}+y^{2} \mathbf{X}_{2}^{\prime}+\ldots+y^{2-2} \mathbf{X}_{n-2}^{\prime}+y^{n-1} \mathbf{X}_{n-1}+\frac{\mathrm{d} U}{\mathrm{~d} x} \\
& =\mathrm{X}_{\mathrm{I}}+2 y \mathbf{X}_{2}+3 y^{2} \mathbf{X}_{3}+\ldots+(n-1) y^{n-2} \mathbf{X}_{n-1}+\frac{\mathrm{d} U}{\mathrm{~d} y} .
\end{aligned}
$$

On peut satisfaire identiquement à cetle équation en faisant:

$$
\mathrm{X}_{1}=\mathrm{X}_{\mathrm{o}}^{\prime} ; \quad 2 \mathrm{X}_{2}=\mathrm{X}_{\mathrm{1}}^{\prime} ; \quad 3 \mathrm{X}_{3}=\mathrm{X}_{0}^{\prime} ; \ldots(n-1) \mathrm{X}_{n-1}=\mathrm{X}_{n-2}^{\prime}
$$

et

$$
y^{n-1} \mathrm{X}_{n-1}+\frac{\mathrm{d} \mathrm{U}}{\mathrm{d} x}=\frac{\mathrm{d} \mathrm{U}}{\mathrm{d} y} .
$$

Les équations (1) et (2) ne renferment que $n$ conditions entre $n+1$ quantités a déterminer. Il faut donc pour les déterminer toutes, ajouter encore la condition, que U s'évanouisse, si l'on pose $y=0$. On obtient donc:

$$
\begin{gathered}
\mathrm{X}_{0}=f(x), \quad \mathrm{X}_{1}=f^{\prime}(x) ; \quad \mathrm{X}_{2}=\frac{1}{1.2} f^{\prime \prime}(x) ; \ldots \mathrm{X}_{n-1}=\frac{1}{1.2 \ldots(n-1)} f^{(n-1)}(x) \\
\mathrm{X}_{n-1}^{\prime}=\frac{1}{1.2 .3 \ldots(n-1)} f^{(n)}(x)
\end{gathered}
$$

et par conséquent l'équation (2) devient:

$$
\frac{y^{n-1}}{1.2 \ldots(n-1)} f^{(n)}(x)+\frac{\mathrm{dU}}{\mathrm{d} x}=\frac{\mathrm{dU}}{\mathrm{d} y}
$$

ou, si l'on pose, pour abréger

$$
\mathrm{U}=\frac{y^{n}}{1.2 .3 \ldots n} \cdot u
$$

et remarquant ensuite que

$$
\frac{\mathrm{dU}}{\mathrm{d} x}=\frac{y^{n}}{1.2 .3 \ldots n} \cdot \frac{\mathrm{d} u}{\mathrm{~d} x} ; \quad \frac{\mathrm{dU}}{\mathrm{d} y}=\frac{y^{n-1}}{1.2 \ldots(n-1)} u+\frac{y^{n}}{1.2 \ldots n} \frac{\mathrm{d} u}{\mathrm{~d} y}
$$

nous aurons, comme on voit bien aisément, l'équation

$$
u-f^{(n)}(x)+\frac{y}{n}\left(\frac{\mathrm{d} u}{\mathrm{~d} y}-\frac{\mathrm{d} u}{\mathrm{~d} x}\right)=0 .
$$


2.

L'integration de cetle équation aux differences partielles donnerail immédiatement le reste sous la forme connue d'une integrale definie. Mais on peut trouver la valeur de $u$ d'une manière tout-à-fait différente; en cherchant deux valeurs de $u$, qui produjsent dans l'expression

$$
\mathbf{F}(u)=u-f^{(n)}(x)+\frac{y}{n}\left(\frac{\mathrm{d} u}{\mathrm{~d} y}-\frac{\mathrm{d} u}{\mathrm{~d} x}\right)=0
$$

un changement de signe; car il existera nécessairement entre ces deux valeurs in autre valeur qui saitsfera à l'équation :

$$
\mathbf{F}(u)=\mathbf{0} \text {. }
$$

Substituons donc dans l'équation précédente au lieu de $u$ :

$$
u_{\mathrm{r}}=f^{(n)}(x) \quad \text { on aura } \quad \mathrm{F}\left(u_{\mathrm{x}}\right)=-\frac{y}{n} f^{(n+\mathrm{x})}(x)
$$

et de même

$$
u_{2}=f^{(n)}(x+y) \quad \text { on aura } \quad \mathbf{F}\left(u_{2}\right)=f^{(n)}(x+y)-f^{(\kappa)}(x) .
$$

Quant aux signes de ces deux valeurs de $\mathrm{F}(u)$ il faut distinguer deux cas :

Les expressions

$$
f^{(n+1)}(x) \quad \text { et } \quad f^{(n)}(x+y)-f^{(n)}(x)
$$

peuvent avoir des signes égaux on opposés.

Dans le cas premier, si les signes sont égaux, les signes de $\mathrm{F}\left(u_{\mathrm{s}}\right)$ el de $\mathrm{F}\left(u_{2}\right)$ sont opposés. Supposons donc que la fonction $f^{(n)}(x)$ soit finie et continue entre les valeurs $x$ et $x+y$ de la variable: il faut nécessairement qu'entre

$$
u_{1}=f^{(n)}(x) \quad \text { et } \quad u_{2}=f^{(n)}(x+y)
$$

se trouve une valeur de $u$, qui fasse évanouir la fonction $\mathrm{F}(u)$. Par conséquent on peut déterminer la valeur d'une quantité $x$ entre 0 et +1 en sorte que

$$
u=f^{(n)}(x+\times y) \quad \text { et } \quad \mathrm{F}(u)=0 .
$$

$\mathrm{Si}$, dans le second cas, les signes des expressions

$$
f^{(n+1)}(x) \quad \text { et } \quad f^{(n)}(x+y)-f^{(n)}(x)
$$

sont opposés, il est clair que $f^{(n+1)}(x)$ entre les deux valeurs $x$ er $x+y$ de la variable changera au moins une fois le signe (v. A.) et par conséquent, qu'elle passera dans cet intervalle, une fois au moins, du croissement au décroissement.

3.

Cela posé, soient $x_{0}, x_{1}, x_{2}, \ldots$ les valeurs constantes de $x$, contenues dans les limites $x$ et $x+y$, qui font que 


$$
f^{(n+1)}(x)=0
$$

et ces valeurs soient ordonnées de manière, qu'on ait $x<x_{0}<x_{1}<\ldots<x+y$.

Si l'on substitue

$$
u_{0}=f^{(n)}\left(x_{0}\right), \quad \text { on aura } \quad \mathrm{F}\left(u_{0}\right)=f^{(n)}\left(x_{0}\right)-f^{(n)}(x)
$$

puisque $x_{0}$ est constante et indépendante de $x$ et $y$. Or $x_{0}$ est la plus petite de toutes les valeurs qui sont au-dessus de $x$, et qui font disparaitre $f^{(n+1)}(x)$; il faut donc que les fonctions :

$$
f^{(n+1)}(x) \quad \text { el } \quad f^{(n)}\left(x_{0}\right)-f_{i x_{j}}^{(n)}=\mathbf{F}\left(u_{0}\right)
$$

aient les mèmes signes (v. A.) : on conclut de là que :

$$
\mathbf{F}\left(u_{0}\right) \quad \text { et } \quad \mathbf{F}\left(u_{2}\right)
$$

ont aussi des signes opposés, d'où il suil qu'il y a entre

$$
u_{0}=f^{(n)}\left(x_{0}\right) \quad \text { et } \quad u_{2}=f^{(n)}(x+y)
$$

une valeur de $u$, et de mème entre $x_{0}$ et $x+y$ une valeur correspondante de $x$, qui fait $F(u)=0$.

Mais il n'en est pas moins sûr que, si cette valeur de $x$ est entre $x_{0}$ et $x+y$, elle se trouvera aussi dans l'intervalle amplifié de $x$ jusqu'à $x+y$, el qu'on peut la représenter de même par $x+x y$, d'où il suit que

$$
u=f^{(n)}(x+x y)
$$

comme dans l'art. 2. - On voil de cette discussion, que ces deux cas, séparés au commencement, peuvent ètre compris dans un même résultat.

Sans supposer finies et continues (v. C.) les fonctions $f(x)$ et $f^{(n)}(x)$ dans l'intervalle de $x$ à $x+y$ de la variable, les conclusions précédentes ne seraient pas valables. Cette supposition n'aurait pas lieu (v. B.) si les fonctions $f^{(n+1)}(x), f^{(n-2)}(x)$, $\ldots f^{\prime}(x), f(x)$ ne restaient pas finies et continues dans l'intervalle marqué.

Résumant tont cela, nous aurons ce Théorème :

Si les fonctions $f(x), f^{\prime}(x), \ldots f^{(n-1)}(x), f^{(n)}(x)$ sont finies et continues pour toute valeur de la variable entre $x$ et $x+y$, et si l'on désigne par $x$ une fraction comprise entre les limites 0 et +1 , et déterminée de manière que

$$
u=f^{(n)}(x+x y) \quad \text { satisfasse à l'équation } u-f^{(n)}(x)+\frac{y}{n}\left(\frac{\mathrm{d} u}{\mathrm{~d} y}-\frac{\mathrm{d} u}{\mathrm{~d} x}\right)=0
$$

on a toujours :

$$
f(x+y)=f(x)+\frac{y}{1} f^{\prime}(x)+\frac{y^{2}}{1.2} f^{\prime \prime}(x)+\cdots+\frac{y^{n-1}}{1.2 . .(n-1)} f^{(n-1)}(x)+\frac{y^{n}}{1.2 .3 . . n} f^{(n)}(x+x y) .
$$

Q. e. d.

Gratz. 1859. 